2020-12-25

\title{
Defining the Syrian hamster as a highly susceptible preclinical model for SARS-CoV-2 infection
}

\section{Rosenke, $\mathrm{K}$}

http://hdl.handle.net/10026.1/17499

\subsection{0/22221751.2020.1858177}

Emerging Microbes \& Infections

Informa UK Limited

All content in PEARL is protected by copyright law. Author manuscripts are made available in accordance with publisher policies. Please cite only the published version using the details provided on the item record or document. In the absence of an open licence (e.g. Creative Commons), permissions for further reuse of content should be sought from the publisher or author. 


\title{
Defining the Syrian hamster as a highly susceptible preclinical model for SARS-CoV-2 infection
}

\author{
Kyle Rosenke ${ }^{a}$, Kimberly Meade-White ${ }^{a}$, Michael Letko $^{a *}$, Chad Clancy $^{b}$, Frederick Hansen ${ }^{a}$, Yanan Liuc, \\ Atsushi Okumura ${ }^{a}$, Tsing-Lee Tang-Huau ${ }^{a}$, Rong $\mathrm{Li}^{\mathrm{c}}$, Greg Saturday ${ }^{\mathrm{b}}$, Friederike Feldmann ${ }^{\mathrm{b}}$, Dana Scott ${ }^{\mathrm{b}}$, \\ Zhongde Wang ${ }^{c}$, Vincent Munster (10 a , Michael A. Jarvis ${ }^{\mathrm{a}, \mathrm{d}}$ and Heinz Feldmann (1) ${ }^{\mathrm{a}}$ \\ aLaboratory of Virology, Division of Intramural Research, National Institute of Allergy and Infectious Diseases, National Institutes of Health, \\ Hamilton, MT, USA; ${ }^{\mathrm{b}}$ Rocky Mountain Veterinary Branch, Division of Intramural Research, National Institute of Allergy and Infectious \\ Diseases, National Institutes of Health, Hamilton, MT, USA; 'Department of Animal, Dairy, and Veterinary Sciences, Utah State University, \\ Logan, UT, USA; 'University of Plymouth and The Vaccine Group Ltd, Plymouth, Devon, UK
}

\section{ABSTRACT}

Following emergence in late 2019, SARS-CoV-2 rapidly became pandemic and is presently responsible for millions of infections and hundreds of thousands of deaths worldwide. There is currently no approved vaccine to halt the spread of SARS-CoV-2 and only very few treatment options are available to manage COVID-19 patients. For development of preclinical countermeasures, reliable and well-characterized small animal disease models will be of paramount importance. Here we show that intranasal inoculation of SARS-CoV-2 into Syrian hamsters consistently caused moderate broncho-interstitial pneumonia, with high viral lung loads and extensive virus shedding, but animals only displayed transient mild disease. We determined the infectious dose 50 to be only five infectious particles, making the Syrian hamster a highly susceptible model for SARS-CoV-2 infection. Neither hamster age nor sex had any impact on the severity of disease or course of infection. Finally, prolonged viral persistence in interleukin 2 receptor gamma chain knockout hamsters revealed susceptibility of SARS-CoV-2 to adaptive immune control. In conclusion, the Syrian hamster is highly susceptible to SARS-CoV-2 making it a very suitable infection model for COVID-19 countermeasure development.

ARTICLE HISTORY Received 27 September 2020; Revised 20 November 2020; Accepted 26 November 2020

KEYWORDS SARS-CoV-2; hamster; infection model; susceptible; pneumonia

\section{Introduction}

Since emergence of SARS-CoV-2 in late 2019, the virus has spread across the globe causing $>56$ million confirmed infections resulting in over 1,300,000 deaths [1]. SARS-CoV-2 causes coronavirus disease (COVID)-19, which is associated with a broad range of symptoms. These symptoms are most commonly fever, dry cough and fatigue, but can also include myalgia, headache, loss of taste or smell, sore throat, congestion, runny nose, nausea and diarrohea. The incubation period of SARS-CoV-2 ranges from 2-14 days with 56 days being most common [2]. While the majority of infections are asymptomatic or present as mild to moderate cases, a small percentage of patients will progress into acute respiratory disease with fatal outcome $[3,4]$. In the absence of a licensed vaccine and only limited treatment options available, scientific and health care communities continue their efforts to rapidly find effective countermeasures for SARS-CoV-2 infections. Aside from new drug or vaccine development, repurposing and "off-label" use of existing FDA-approved compounds is being heavily pursued, often omitting preclinical animal studies before moving directly into humans [5-8].

Preclinical animal models are integral to evaluating countermeasures for infectious diseases such as COVID-19. Non-human primate (NHP) COVID-19 models have been established, and several Old-World monkey species have been shown to be susceptible to SARS-CoV-2 infection. Infection in these animals results in a transient mild to moderate interstitial pneumonia, rather than the severe clinical outcomes [9-11]. Ferrets have also been shown to be susceptible to SARSCoV-2 infection resulting in mild disease with shedding from the upper respiratory tract. Efficient transmission has been documented suggesting the ferret may be a valuable preclinical model for transmission but not for severe disease [12-14].

Development of small animal SARS-CoV-2 infection models was initially delayed, but recently both mouse and hamsters COVID-19 models have been

CONTACT Heinz Feldmann feldmannh@niaid.nih.gov E Laboratory of Virology, National Institute of Allergy and Infectious Diseases, National Institutes of Health, 903 S 4th Street, Hamilton, MT US-59840, USA

*Current affiliation: Paul G. Allen School for Global Animal Health, Washington State University, Pullman, WA, USA.

(4) Supplemental data for this article can be accessed at https://doi.org/10.1080/22221751.2020.1858177

( 2020 The Author(s). Published by Informa UK Limited, trading as Taylor \& Francis Group, on behalf of Shanghai Shangyixun Cultural Communication Co., Ltd This is an Open Access article distributed under the terms of the Creative Commons Attribution License (http://creativecommons.org/licenses/by/4.0/), which permits unrestricted use, distribution, and reproduction in any medium, provided the original work is properly cited. 
described [15-19]. The mouse angiotensin-converting enzyme 2 (ACE-2) receptor has only low affinity for the SARS-CoV-2 spike protein leading to poor binding and entry $[20,21]$. Initially, mouse susceptibility to SARS-CoV-2 infection was increased through transduction of the respiratory tract cells using adenovirus vectors expressing human ACE2, which lead to development of non-lethal pneumonia $[22,23]$. Several receptor transgenic mice have since been created, expressing human ACE2 under tissuespecific promoters or the endogenous mouse ACE2 promoter [16, 24-26]. All of these mice are susceptible to SARS-CoV-2 infection, resulting in a range of clinical signs with mild to fatal disease depending on the transgene. SARS-CoV-2 adaptation to mice has also been attempted through either serial passaging or reverse genetics. So far this approach has sensitized multiple strains of mice to infection in the upper and lower respiratory tracts, leading to very mild disease [15,27].

Syrian hamsters have been reported to be susceptible to SARS-CoV-2 infection developing moderate interstitial pneumonia leading to transient mild to moderate disease $[17,19]$. However, these studies have used varying doses of SARS-CoV-2 for inoculation, and the impact of age and sex on infection and disease is unclear. Herein, we defined the Syrian hamster as a SARS-CoV-2 infection and disease model and refined further virologic and host parameters to increase the value of this small animal model. First, we demonstrated high functional interaction of the SARS-CoV-2 receptor binding domain (RBD) with the hamster ACE2 receptor. Next, we determined the SARS-CoV-2 dose causing infection in $50 \%$ of animals $\left(\mathrm{ID}_{50}\right)$ following intranasal infection, showing these animals to be highly susceptible to infection. We detailed the progression of SARSCoV-2 infection, and also the effect of sex and age on SARS-CoV-2 infection in the model. Finally, we investigated SARS-CoV-2 infection of interleukin 2 receptor, gamma chain $(I L 2 R G)$ knockout hamsters to assess the impact of adaptive (and NK) immunity in this model.

\section{Materials and methods}

Biosafety and ethics. All work using live SARS-COV2 was performed in BSL4 using standard operating protocols approved by the Rocky Mountain Laboratories Institutional Biosafety Committee. All animal work was approved by the Institutional animal Care and use Committee and performed in strict accordance with the recommendations described in the Guide for the Care and Use of Laboratory Animals of the National Institutes of Health, the Office of Animal Welfare, the United States Department of Agriculture in an association for Assessment and
Accreditation of Laboratory Animal Care-Accredited Facility. Animals were group housed in HEPA-filtered cage systems enriched with nesting material. Commercial food and water were available ad libitum.

Virus. SARS-CoV-2 isolate nCoV-WA1-2020 (MN985325.1) was kindly provided by CDC as Vero passage 3 [28]. The virus was propagated once in Vero E6 cells in DMEM (Sigma) supplemented with $2 \%$ foetal bovine serum (Gibco), $1 \mathrm{mM} \mathrm{L}$-glutamine (Gibco), $50 \mathrm{U} / \mathrm{ml}$ penicillin and $50 \mu \mathrm{g} / \mathrm{ml}$ streptomycin (Gibco) (virus isolation medium). Whole genome sequencing of virus stock revealed it was $100 \%$ identical to the initial deposited Genbank sequence (MN985325.1) and no contaminants were detected.

Cells. Vero E6 cells were maintained in DMEM (Sigma) supplemented with $10 \%$ foetal calf serum (Gibco), $1 \mathrm{mM}$ L-glutamine (Gibco), $50 \mathrm{U} / \mathrm{ml}$ penicillin and $50 \mu \mathrm{g} / \mathrm{ml}$ streptomycin (Gibco). 293T and baby hamster kidney (BHK) were maintained in DMEM (Gibco) supplemented with foetal bovine serum, penicillin/streptomycin and L-glutamine.

Plasmids. SARS-CoV-2 spike protein plasmids were previously described [20]. Sequences from SARS-CoV-1/Urbani spike (GenBank MN908947), SARS-CoV-2 spike RBD (AY278741), human ACE2 (GenBank Q9BYF1.2) and hamster ACE2 (GenBank: XP_005074266.1) were codon optimized for humans and cloned into pcDNA3.1 + .

Cell entry assay. Vesicular stomatitis virus (VSV) particles were pseudotyped with different wildtype or chimeric spike proteins or no spike in $293 \mathrm{~T}$ cells as previously described [20]. BHK cells were transfected in 96-well format with $100 \mathrm{ng}$ of host receptor plasmid, or no receptor and subsequently infected with spike-pseudotyped VSV particles as previously described [20]. Approximately $18 \mathrm{~h}$ later, luciferase was measured using the BrightGlo reagent (Promega), following the manufacturer's instructions.

Animal studies. Syrian hamsters (Mesocricetus auratus), 6-8-weeks-of-age and >27-weeks-of-age, males and females, were purchased from Envigo. IL2RG KO hamsters were generated with CRISPR/ Cas9-mediated gene targeting technique established in the hamster by Utah State University as reported previously [29]. Hamsters were anesthetized by inhalation of vaporized isoflurane and inoculated via intranasal instillation with $50 \mu \mathrm{l}$ of varying concentrations of inoculum $\left(1-1 \times 10^{5}\right.$ tissue culture dose 50 $\left.\left(\mathrm{TCID}_{50}\right)\right)$ dropped into each naris $(25 \mathrm{ul} /$ naris $)$ using a pipette. Hamsters were weighed and monitored daily for signs of disease. Temperature transponders (BMDS IPTT-300) were implanted subcutaneously under anesthesia just above the shoulder blades in a subset of hamsters to monitor and record temperatures daily. Swabs (oral and rectal) were taken at different days post-infection using polyester flock tipped swabs (Puritan Medical Products). Animals 
were euthanized for necropsies at different timepoints to assess disease.

Virus titration. Virus was quantified through endpoint titrations performed in Vero E6 cells. Tissue was homogenized in $1 \mathrm{ml}$ DMEM using a TissueLyzer (Qiagen) and centrifuged to remove cellular debris (10 $\mathrm{min}$ at $8,000 \mathrm{rpm})$. Cells were inoculated with 10 -fold serial dilutions of clarified tissue homogenate or whole blood samples in $100 \mu \mathrm{l}$ DMEM (SigmaAldrich) supplemented with $2 \%$ foetal bovine serum, $1 \mathrm{mM}$ L-glutamine, $50 \mathrm{U} / \mathrm{ml}$ penicillin and $50 \mu \mathrm{g} / \mathrm{ml}$ streptomycin. Cells were incubated for seven days and then scored for cytopathic effect (CPE). The $\mathrm{TCID}_{50}$ was calculated via the Reed-Muench formula [30].

Viral genome load. qRT-PCR was performed on RNA extracted from blood and swabs using QiaAmp Viral RNA kit (Qiagen) according to the manufacturer's instructions. Tissues ( $\leq 30 \mathrm{mg}$ ) were homogenized in RLT buffer and RNA was extracted using the RNeasy kit (Qiagen) according to the manufacturer protocol. Viral genomic RNA (gRNA) was detected with a one-step real-time RT-PCR assay (Quantifast, Qiagen) using primers and probes generated to target either the SARS-CoV-2 E [31] or N gene (forward: 5'AGAATGGAGAACGCAGTGGG; reverse: 5'- TGAGAGCGGTGAACCAAGAC; probe: 5'-CGATCAAAACAACGTCGGCC synthesized with 5' 6carboxyfluorescein, internal Zen quencher and 3' Iowa black quencher); all primers and probes were synthesized by Integrated DNA Technologies (IDT). Dilutions of RNA standards quantified by droplet digital PCR were run in parallel and used to calculate gRNA copies with the E assay. The N-based assay used a standard curve synthesized as follows: T7 in vitro transcription (ThermoFisher) of a synthetically produced $\mathrm{N}$ sequence (IDT) was used to generate template RNA. RNA was quantified by $260 \mathrm{~nm}$ absorbance to determine copy number and a standard curve generated by serial dilution.

Histopathology and immunohistochemistry. Tissues were fixed in $10 \%$ neutral buffered formalin (with two changes) for a minimum of 7 days. Tissues were placed in cassettes and processed with a Sakura VIP-6 Tissue Tek on a 12-hour automated schedule, using a graded series of ethanol, xylene, and ParaPlast Extra. Embedded tissues are sectioned at $5 \mathrm{um}$ and dried overnight at $42^{\circ} \mathrm{C}$ prior to staining. Specific anti-CoV immunoreactivity was detected using GenScript U864YFA140-4/ CB2093 NP-1 at a 1:1,000 dilution. The secondary antibody is an anti-rabbit IgG polymer from Vector Laboratories ImPress VR. Tissues were then processed for immunohistochemistry using the Discovery Ultra automated processor (Ventana Medical Systems) with a ChromoMap DAB kit (Roche Tissue Diagnostics).
Statistical analyses. Statistical analysis was performed in Prism 8 (GraphPad). T-tests were used to assess studies with 2 groups, ANOVA was used to analyse studies with $>2$ groups.

\section{Results}

The SARS-CoV-2 spike protein binds to the Syrian hamster ACE2 receptor. To validate that that SARS$\mathrm{CoV}-2$ can bind and then use as an entry receptor the Syrian hamster ACE2 receptor, a VSV pseudotype assay was performed as previously published [20]. Briefly, a chimeric SARS-CoV-1 spike protein was generated with the RBD replaced with the SARSCoV-2 RBD (VSV-SARS-CoV-2-RBD), a psuedotype that has previously shown comparable binding to ACE-2 as the WT [20,32]. We transfected BHK cells that do not express ACE2 with expression plasmids for either human or hamster ACE2, or empty vector as a negative control. Cells were then infected with the VSV pseudotyped particles carrying either SARSCoV-1 full-length spike (VSV-SARS-CoV-1-RBD) or chimeric VSV-SARS-CoV-2-RBD. As anticipated, both VSV-SARS-COV-1-RBD and VSV-SARS-CoV2-RBD were unable to enter $\mathrm{BKH}$ cells, but entry was rescued in transduced cells expressing the hamster or human ACE2. Interestingly, VSV-SARS-CoV-2$\mathrm{RBD}$ entry was increased compared to VSV-SARSCoV-1-RBD, independent of the ACE2 origin, which may indicate higher susceptibility of both hamsters and humans to SARS-CoV-2 compared to SARSCoV-1 (Figure 1).

Syrian hamsters are highly susceptible to SARSCoV-2. To determine the level of susceptibility of

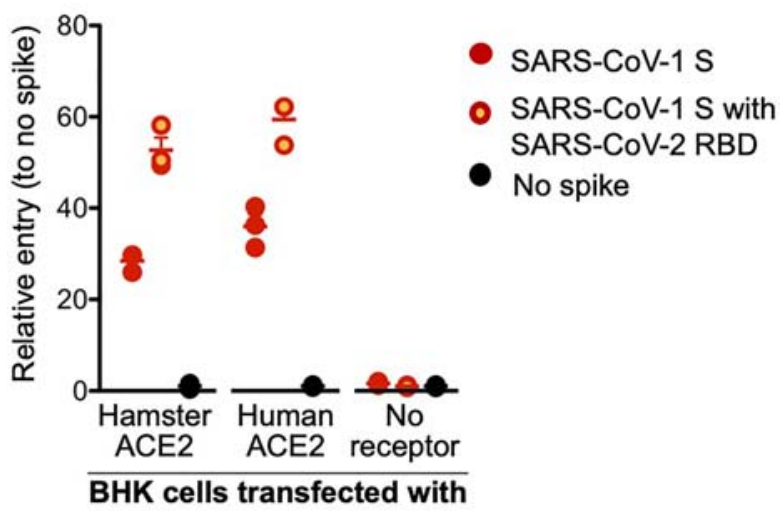

Figure 1. SARS-CoV-2 spike receptor binding data. A VSV pseudotype assay was used to assess the binding affinity of the SARS-CoV-2 RBD. BHK cells expressing either the human or Syrian hamster or no ACE2 receptor were infected with VSV-pseudotyped particles carrying either the SARS-CoV-1 spike (S) protein or a chimeric SARS-CoV-1 spike with the SARS-CoV-2 receptor binding domain (RBD). Note: red circles, SARS-CoV-1 S; yellow circles with red outline, SARS-CoV-1 S with SARS-CoV-2 RBD; black circle, no ACE-2; S, spike protein; $R B D$, receptor binding domain. 
Syrian hamsters to SARS-CoV-2 infection, four groups of six hamsters aged 4-6 weeks were infected with limiting dilutions of SARS-CoV-2 to determine the $\mathrm{ID}_{50}$. Groups were intranasally infected with a ten-fold serial dilution series of virus ranging from $10^{3}$ to $10^{0} \mathrm{TCID}_{50}$, and infection course was monitored by signs of disease including weight and temperature. Although no significant changes in temperature over the experimental period were observed, weight loss between the animal groups directly correlated with the infectious dose (Figure 2 (A)). Oral and rectal swabs were taken at 3 days post infection (dpi) and 5dpi to measure differences in levels of viral gRNA between the different groups. Only one animal in the group receiving the 1 $\mathrm{TCID}_{50}$ dose had detectable gRNA in the oral swabs and none in the rectal swab (Figure 2(B)), with only half the animals in this group being positive at $5 \mathrm{dpi}$ (Figure 2(C)). Lungs were harvested at 5dpi and gRNA and infectious titres were determined. Remarkably similar gRNA levels were found across groups infected with $10 \mathrm{TCID}_{50}$ or higher, only the 1 TCID $_{50}$ group had a significant reduction in gRNA levels (Figure 2(D)). Infectious titres from the lungs of each dose group had a similar pattern with high viral loads in all groups, except the $1 \mathrm{TCID}_{50}$ group for which no infectious virus was detected (Figure 2 (E)). Remarkably, this series of experiments shows the $\mathrm{ID}_{50}$ in Syrian hamsters to be only $5 \mathrm{TCID}_{50}$ when administered intranasally.

SARS-CoV-2 infection of Syrian hamsters results in broncho-interstitial pneumonia. To characterize the extent of disease in this model, two groups of 10 Syrian hamsters aged 4-6 weeks were intranasally infected with either $500 \mathrm{ID}_{50}$ (low dose; $10^{3} \mathrm{TCID}_{50}$ ) or $5 \times 10^{4} \mathrm{ID}_{50}$ (high dose; $10^{5} \mathrm{TCID}_{50}$ ) of SARS$\mathrm{CoV}-2$. Animals were monitored for clinical symptoms of disease with the intent of euthanizing a subset of animals for analysis when early symptoms became apparent. At 3dpi hamsters in both groups had lost weight on consecutive days (Figure 3(A)) and displayed slightly ruffled fur with minor changes in respiration pattern. Four animals from each group were euthanized at this time (3dpi) for analysis. Lungs were examined for gross pathology and all animals had lung lesions consisting of focal extensive areas of pulmonary edema and consolidation with evidence of interstitial pneumonia characterized by a failure of the lungs to collapse following removal. No gross pathology was observed in other tissues collected including liver, spleen, kidney and brain. The remaining hamsters continued to lose weight until 5dpi with a maximum loss of $<10 \%$ body weight (Figure $3(\mathrm{~A})$ ); clinical signs remained similar until 5dpi. Three more animals from each group were euthanized at this time (5dpi). At necropsy, an increase in both number and severity of lesions were observed in animals receiving the low dose, but gross pathology was similar in appearance at 3dpi and 5dpi in animals receiving the high dose. Following four consecutive days of weight gain (Figure 3(A)) and improving clinical signs, the remaining animals from each group were euthanized at 10dpi. Gross examination of the lungs at 10dpi showed a significant reduction in both lesion severity and congestion relative to lungs taken earlier during disease progression. Despite the obvious development of respiratory disease at earlier times postinfection, clinical signs were minimal at this stage (10dpi) with animals having recovered weight (Figure $3(\mathrm{~A})$ ). Oral and rectal swabs were collected at each time point to monitor viral gRNA shedding. Shedding peaked in all swab types at $3 \mathrm{dpi}$ with a small decrease at $5 \mathrm{dpi}$ before dropping significantly at 10dpi (Figure 3 $(B, C))$. Lungs were evaluated for both SARS-CoV-2 gRNA and infectious titres. gRNA loads in the lungs were high with $>10^{10}$ genome copies/gram (Figure 3 (D)), whereas gRNA loads in other organs were approximately $4-5$ logs lower (Sup. Figure 1). Lung infectious titres were $10^{7} \mathrm{TCID}_{50}$ per gram at $3 \mathrm{dpi}$ although this had already decreased at 5dpi and was absent in all but one animal at 10dpi (Figure 3(E)). Overall, there was no significant difference between the groups infected with the low and high dose of SARS-CoV-2, except for 10dpi where one animal remained positive.

ACE2 immunoreactivity is observed in the convoluted tubules of the kidney and liver sinusoids but is not routinely detected in the trachea or lung [33]. Interestingly, no significant histopathologic lesions were observed in the evaluated sections of liver, kidney and spleen of any animal regardless of age, sex or inoculation dose used in these studies. Immunohistochemistry also failed to reveal SARS-CoV-2 antigen in the liver, kidney and spleens examined. Pathologically, changes associated with disease in the lower respiratory tract were noted in both the trachea and lung regardless of the inoculation dose. The observed pathology had a more distinct progression in the low $\left(500 \mathrm{ID}_{50}\right)$ inoculation dose than the higher dosed group $\left(5 \times 10^{4} \mathrm{ID}_{50}\right)$. Evidence of bronchointerstitial pneumonia was observed at all evaluated time points. At $3 \mathrm{dpi}$, lesions were characterized by epithelial necrosis in the trachea and bronchioles, squamous metaplasia of the mucosa in the trachea, bronchiolitis characterized by influx of neutrophils and macrophages into the lamina propria and mild interstitial pneumonia with expansion of alveolar septa by edema fluid, with few strands of fibrin and low numbers of leukocytes (Figure 4(A-C)). By $5 \mathrm{dpi}$, the interstitial pneumonia was moderate to severe with fibrin leaking into alveolar spaces, alveolar edema, influx of moderate numbers to numerous neutrophils and macrophages into alveolar spaces, presence of syncytial cells in bronchioles and alveolar 
A

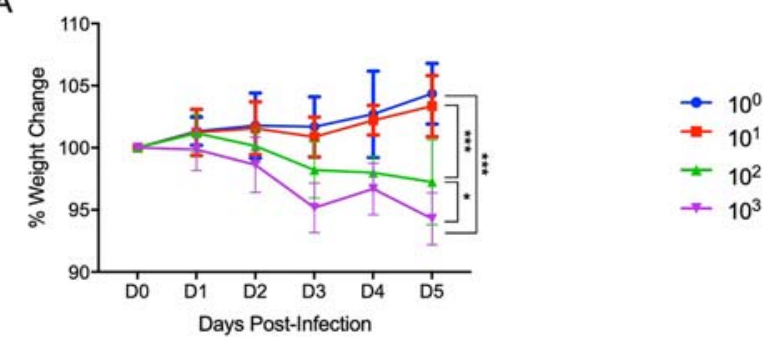

$\mathrm{B}$

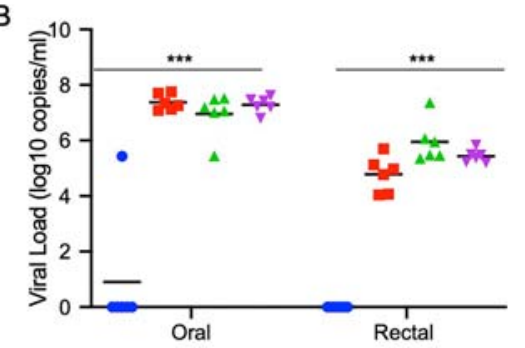

D

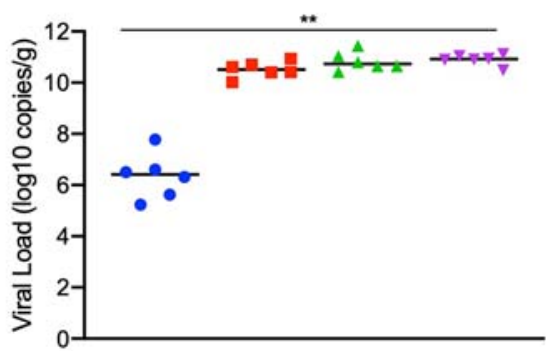

C

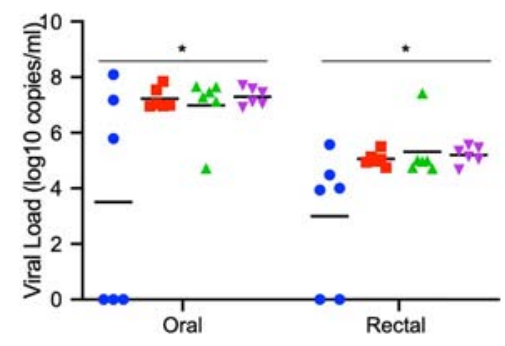

E

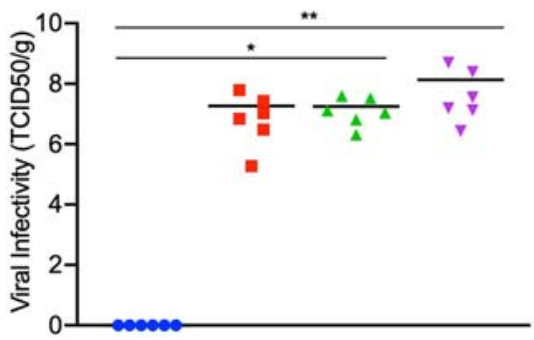

Figure 2. Susceptibility of Syrian hamsters to SARS-CoV-2. Syrian hamsters were inoculated intranasally with 10-fold limiting dilutions of SARS-CoV-2 beginning at $10^{3} \mathrm{TCID}_{50}$. Weights were collected daily and shedding was assessed via swab samples (nasal and rectal) collected at 3 and 5 dpi. Viral loads were determined as genome copies and infectious virus. (A) Daily weights. (B) Shedding at $3 \mathrm{dpi}$. (C) Shedding at $5 \mathrm{dpi}$. (D) Viral genome load in the lungs at $5 \mathrm{dpi}$. (E) Infectious lung titres at $5 \mathrm{dpi}$. A statistical significance was found between the groups presented in $(A)$, with the group receiving the highest dose of $10^{3} \operatorname{TCI}_{50}$ losing the most weight. The group receiving the second highest infectious dose $\left(10^{2} \mathrm{TCID}_{50}\right)$ lost statistically less than the $10^{3} \mathrm{TCID}_{50}$ group but statistically more weight than the 2 groups receiving the two lowest infectious doses. (B-E) A statistically significance difference was found between the group receiving the lowest dose $\left(10^{\circ} \mathrm{TCI} \mathrm{D}_{50}\right)$ and all other groups. Multiple $t$ tests comparing groups directly were used to analyse significance. Note: blue circles, $10^{0} \mathrm{TCID}_{50}$ dose; red square, $10^{1} \mathrm{TCID}_{50}$ dose; green triangle, $10^{2}$ $\mathrm{TCID}_{50}$ dose; purple triangle, $10^{3} \mathrm{TCID}_{50}$ dose.

spaces and prominent type II pneumocyte hyperplasia (Figure 4(D-F)). Evidence of lesion resolution was observed at 10dpi with a decrease in alveolar cellular exudate, absence of epithelial necrosis and a prominent "honeycombing" pattern of type II pneumocyte hyperplasia centred on terminal bronchioles with septal expansion by a small to moderate amount of fibrosis (Figure $4(\mathrm{G}-\mathrm{I})$ ) as compared to naïve infected control animals (Figure 4(J-L)). Mild and multifocal pleural fibrosis was observed at 10dpi (Figure 5). At both 5dpi and 10dpi, moderate numbers of blood vessels were surrounded by perivascular infiltrates of lymphocytes that frequently formed distinct perivascular cuffs, occasionally focally disrupting the tunica media or forming aggregates between the tunica intima and media elevating the endothelium into the lumen. Immunohistochemical reaction demonstrated viral antigen in bronchiole epithelial cells at 3dpi (Figure 5(A-C)) with fewer cells showing immunoreactivity at 5dpi (Figure 5 (D-E)) and no epithelial cells exhibiting immunoreactivity at 10dpi (Figure 5(G-I)). Lower in the respiratory tree, SARS-CoV-2 immunoreactivity was demonstrated in type I and II pneumocytes as well as alveolar macrophages at $3 \mathrm{dpi}$ and $5 \mathrm{dpi}$ with a lack of immunoreactivity at 10dpi (Figure 5(G-I)) which is comparable to uninfected control tissue (Figure 5(J-L)) Overall, pathologic changes progressed more rapidly in animals infected with the high dose relative to the low dose, but severity of disease was consistent between the groups with all animals developing moderate to severe bronchointerstitial pneumonia, commensurate with a mild to moderate infection model.

Neither age nor gender affected disease severity or outcome. To determine whether age or gender would affect disease progression following SARSCoV-2 infection, we infected both young (4-6 weeks 
A

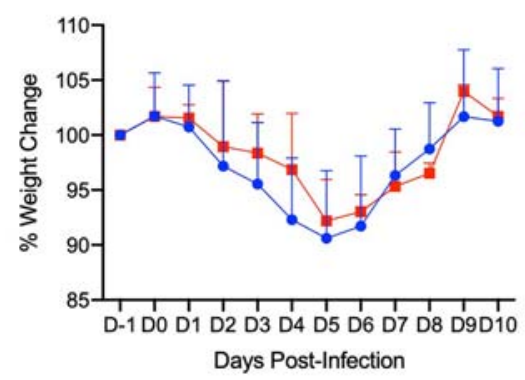

B

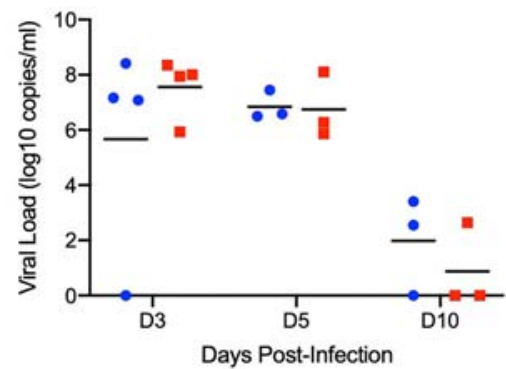

D

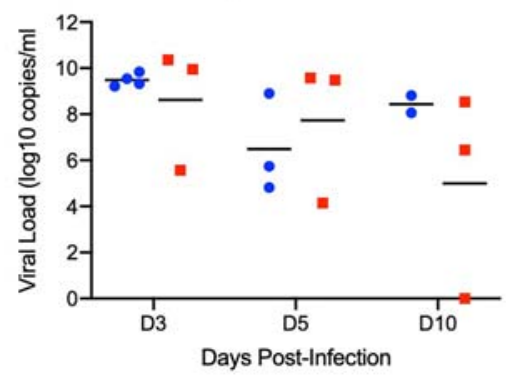

- $10^{5}$

- $10^{3}$
C

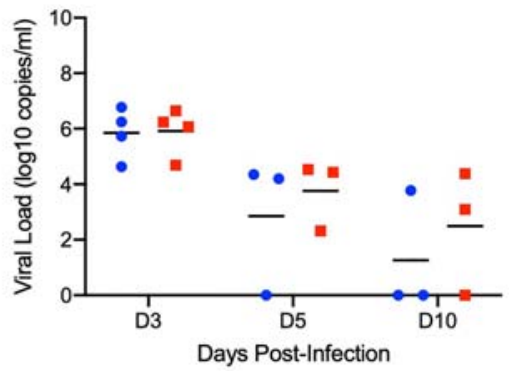

E

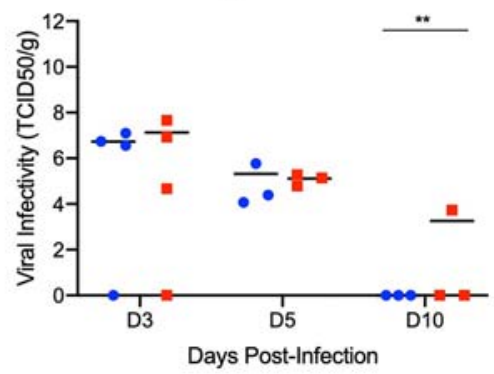

Figure 3. Increased infectious dose does not affect shedding or disease severity. Syrian hamsters were infected intranasally with either $500 I_{50}\left(10^{3} \mathrm{TCID}_{50}\right)$ or $5 \times 10^{4} \mathrm{ID}_{50}\left(10^{5} \mathrm{TCID}_{50}\right)$ of SARS-CoV-2. Samples were collected at the time points noted. Weight were collected daily, shedding from mucosal membranes and viral genome load and infectivity in the lungs were measured. (A) Daily weights. (B) Viral genome load recovered from nasal swabs. (C) Viral genome load recovered from rectal swabs. (D) Viral genome load in the lungs. (E) Infectious titres in the lungs. T-tests were used to compare the two groups at each time where samples were collected. A significant difference was observed at $10 \mathrm{dpi}$ in the lung titres (E), but no other significant differences were observed in this study. Note: blue circles, $10^{5} \mathrm{TCID}_{50}$ dose; red square, $10^{3} \mathrm{TCID}_{50}$ dose.

old) and aged ( $>27$ weeks old) male and female hamsters with the low dose of $500 \operatorname{ID}_{50}\left(10^{3} \mathrm{TCID}_{50}\right)$ by the intranasal route. Consistent with previous studies, animals began to show mild clinical signs of disease and weight loss peaking at 5dpi or 6dpi (Figure 6(A)). A subset of animals in each age group and gender were euthanized for analysis at 3, 5 and 11dpi. Lung pathology was comparable in all groups at each time point throughout the study. Gross lung lesions were evident at $3 \mathrm{dpi}$, had worsened by $5 \mathrm{dpi}$ before mostly resolving at 11dpi (Supp. Figure 2A). To help determine disease severity, lung weights were recorded and calculated as a percentage of overall body weight for comparison. Lungs weights paralleled the observed lesions and were significantly increased at 5dpi (Supp. Figure 2B). These observations were consistent with the development of pneumonia and were independent of sex and age.

High levels of viral gRNA were detected in oral and rectal swabs in all groups. The highest gRNA levels detected were $3 \mathrm{dpi}$ before decreasing at $5 \mathrm{dpi}$ and again at $11 \mathrm{dpi}$ where only a subset remained positive
(Figure 6(B,C)). Interestingly, all oral swabs were positive at 11dpi suggesting viral replication was still ongoing in the upper respiratory areas (Figure 6(B)). Several tissues including blood were collected and examined by qRT-PCR for viral gRNA loads at each timepoint. The lungs had high viral loads with gRNA levels highest 3dpi before decreasing at 5dpi and again at 11dpi (Figure 6(D)). This corresponded with infectious titres which followed a similar pattern and peaked at 3dpi (Figure 6(E)). The brain consistently had the second highest levels of viral gRNA detected and remained relatively stable across the groups at $>10^{8} \mathrm{TCID}_{50}$ equivalents at the time points examined. The digestive tract, both upper and lower, exhibited levels of gRNA of $>10^{6} \mathrm{TCID}_{50}$ equivalents across the study. Liver, spleen and kidneys all had similar levels of gRNA at 3dpi and 5dpi with $>10^{4}$ $\mathrm{TCID}_{50}$ equivalents before decreasing at $11 \mathrm{dpi}$.

Hamsters lacking interleukin-2 receptor subunit gamma (IL2RG KO) show persistent infection with SARS-CoV-2. IL2RG KO hamsters are unable to develop mature NK cells with compromised 

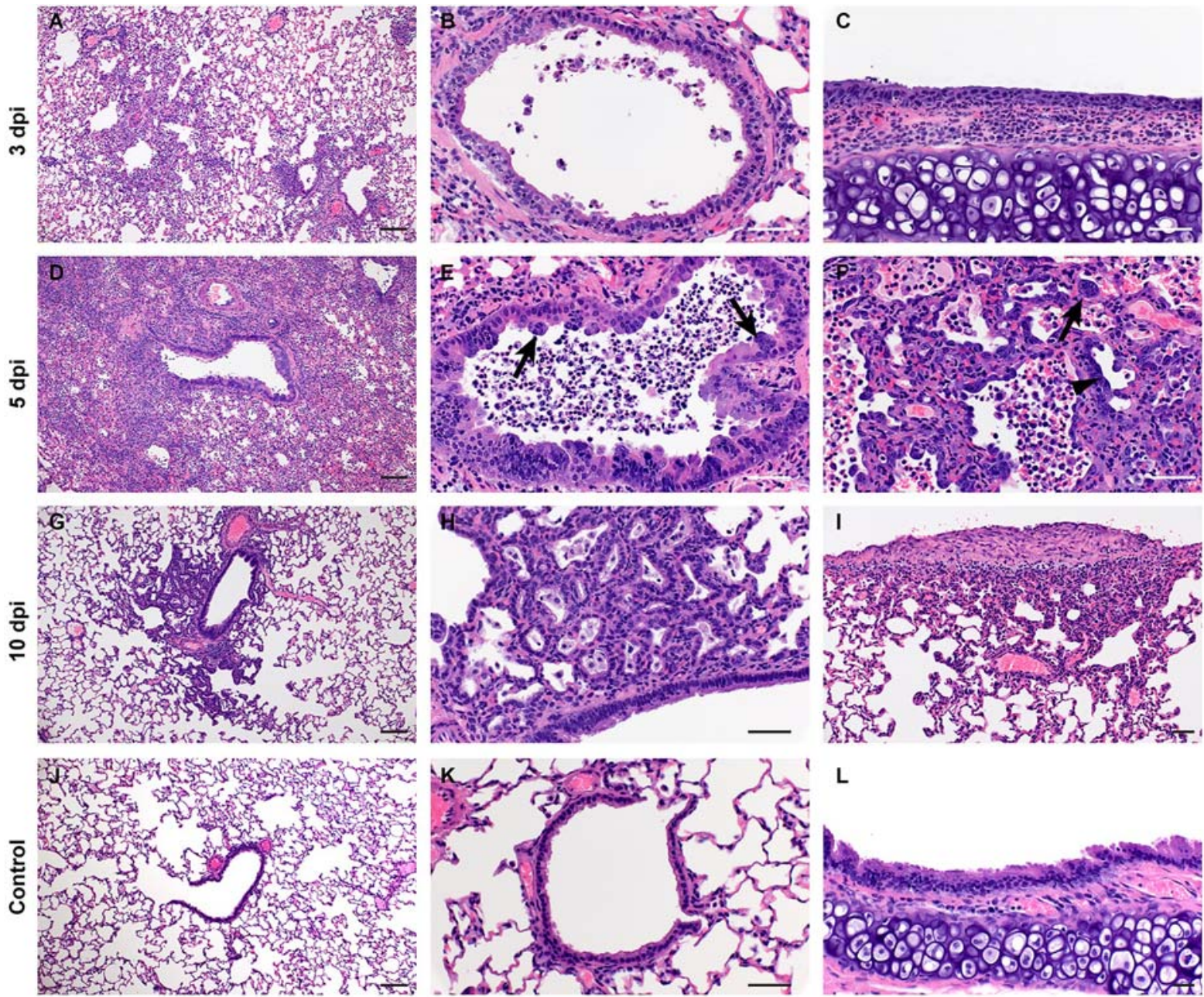

L

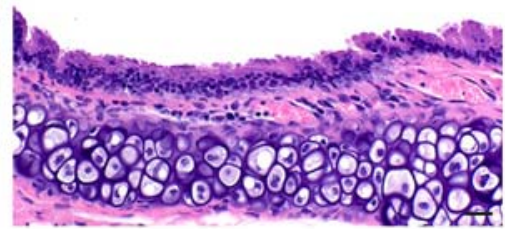

Figure 4. SARS-CoV-2 infection of Syrian hamsters results in broncho-interstitial pneumonia. Syrian hamsters were infected intranasally with $500 \mathrm{ID}_{50}\left(10^{3} \mathrm{TCID}_{50}\right)$ of SARS-CoV-2. Lungs were fixed in $10 \%$ formalin, cut and stained with Hematoxylin and Eosin (HE) to examine pulmonary pathology at 3,5 and $10 \mathrm{dpi}$. (A-C), 3 dpi. (A) Inflammation initiates within interstitial spaces in and around terminal airways with a minimal cellular exudate into the airway spaces (100x, size bar is 50um). (B) Bronchiolar epithelial necrosis with influx of neutrophils into the mucosa and airway lumen (400x, size bar is 20um). (C) Attenuation of the tracheal mucosa with loss of apical cilia accompanied with an influx of moderate numbers of degenerate and non-degenerate neutrophils (400x, size bar is 20um). (D-F), 5 dpi. (D) Locally extensive inflammation is noted (100x, size bar is $50 u m$ ). (E) Progressive bronchiolitis with degenerate and non-degenerate neutrophils and exudate within the lumen and prominent epithelial syncytial cells (arrows; 400x, size bar is 20um). (F) Alveolar spaces contain macrophages and neutrophils. Alveolar septa are thickened and expanded by fibrin, edema fluid and infiltrating leukocytes and are lined by prominent type II pneumocytes (arrowhead) and rare syncytial cells (arrow; 400x, size bar is 20um). (G-I), $10 \mathrm{dpi}$. (G) Resolving inflammation is largely limited to bronchioles and the adjacent alveolar spaces (100x, size bar is 50um) (H) Alveolar septa are thickened by collagen with lymphocytes and lined by numerous plump type II pneumocytes that surround low numbers of foamy alveolar macrophages (400x, size bar is 20um). (I) Multifocal pleural fibrosis is evident with mild subpleural inflammation (200x, size bar is 20um). (J-L), naïve animals. (J) Normal interstitial spaces (100x, size bar is 50um). (K) Normal bronchiolar epithelium (400x, size bar is $20 \mathrm{um}$ ). (L) Normal tracheal mucosa (400x, size bar is 20um).

development of $\mathrm{T}$ and $\mathrm{B}$ lymphocytes [29,34]. This lack of mature lymphoid cells results in an immunocompromised status known as X-linked severe combined immunodeficiency (XSCID) in humans [35]. To ask the question of whether these key cellular aspects of the innate $(\mathrm{NK})$ and adaptive (B and $\mathrm{T}$ cells) impact SARS-CoV-2 replication and associated disease, we assessed infection in IL2RG KO hamsters. Similar to immunocompetent Syrian hamsters, following infection with $5 \times 10^{4} \quad \mathrm{ID}_{50}$ (high dose; $10^{5}$ $\mathrm{TCID}_{50}$ ) of SARS-CoV-2, four (2 males, 2 females) IL2RG KO hamsters lost approximately $5-10 \%$ of their body weight over the first 5 days following infection before recovering (Figure $7(\mathrm{~A})$ ). Oral and rectal swabs were taken at $5 \mathrm{dpi}$ and $24 \mathrm{dpi}$ to measure shedding. Interestingly, both oral and rectal swabs were positive at both time-points and at very similar levels (Figure 7(B)). All four hamsters were euthanized 24dpi following 2 weeks of consistent weight gain and lungs were examined for disease. At examination, the lungs had lesions similar to the immunocompetent hamsters at $5 \mathrm{dpi}$, were congested and failed to collapse. Remarkably, virus titration performed on lung tissue revealed high infectious titres ranging from 

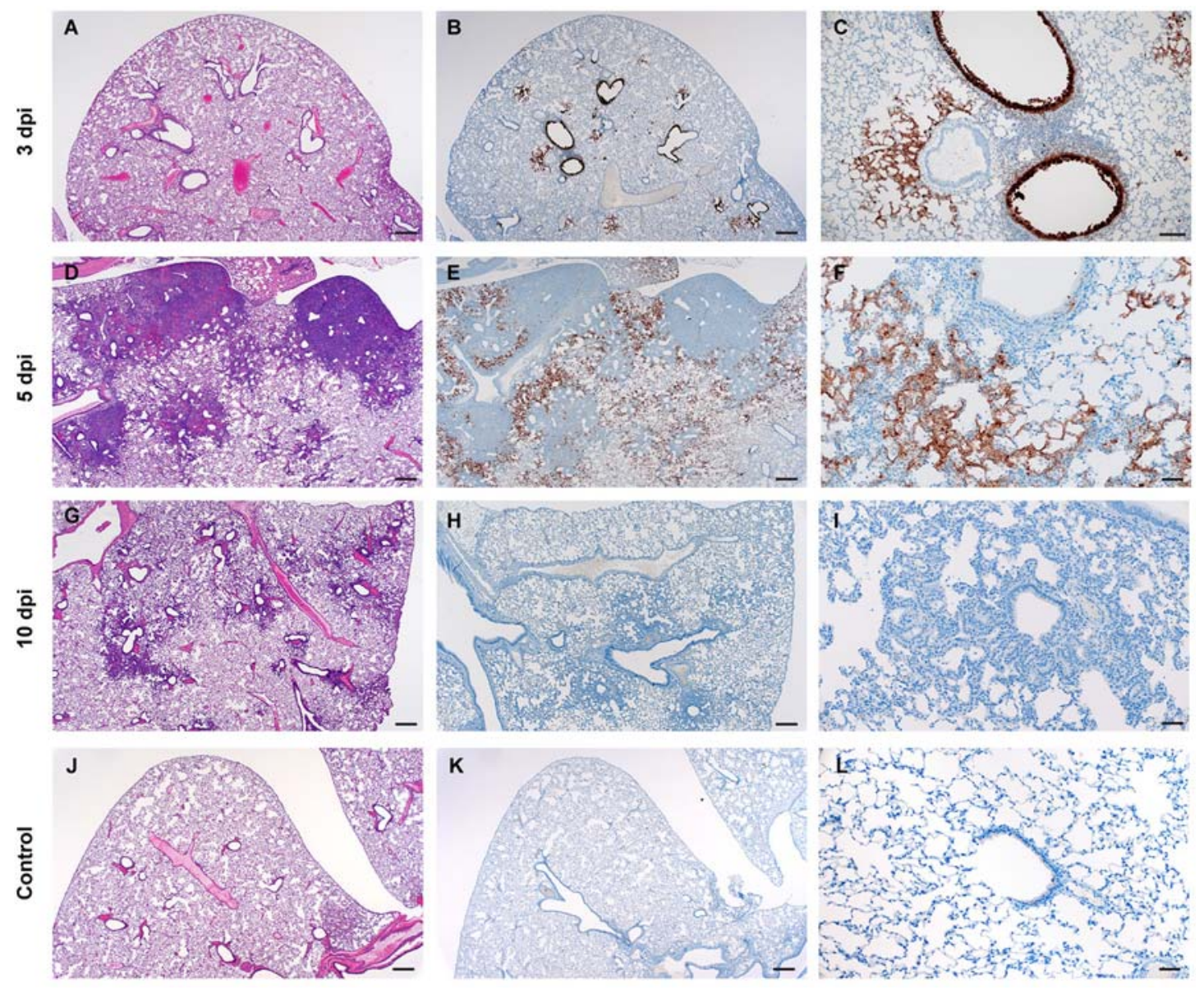

Figure 5. SARS-CoV-2 viral antigen in the lungs over the course of infection. Syrian hamsters were infected intranasally with $500 \mathrm{ID}_{50}\left(10^{3} \mathrm{TCID}_{50}\right)$ of SARS-CoV-2. Histopathology (HE) and immunohistochemistry (IHC) was used to assess pathology with the presence of SARS-CoV-2 antigen in pulmonary sections at 3,5 and $10 \mathrm{dpi}$. (A-C), $3 \mathrm{dpi}$. (A) Histopathology is largely limited to bronchioles and terminal airway spaces and is not readily apparent at a low magnification (H\&E, 20x, size bar is 200um). (B) Immunohistochemical reaction highlights antigen distribution in bronchioles and terminal airway spaces (20x, size bar is 200um). (C) Bronchiolar epithelial cell immunoreactivity with limited antigen detection in alveolar spaces (100x, size bar is 50um). (D-F), 5 dpi. (D) Extension of cellular exudate from bronchioles into alveolar spaces (H\&E, 20x, size bar is 200um). (E) Immunoreactivity is detected along the periphery of regions of pathology and has largely been cleared from bronchiolar epithelium (20x, size bar is 200um). (F) Immunoreactivity is noted in type I and type II pneumocytes and few alveolar macrophages (200x, size bar is 20um). (G-I), $10 \mathrm{dpi}(\mathrm{G})$ Resolving inflammation is limited to bronchioles and adjacent terminal airways (H\&E, 20x, size bar is 200um). (H) SARS-CoV-2 immunoreactivity is not observed in regions of resolving inflammation (20x, size bar is 200um). (I) No immunoreactivity is observed (200x, size bar is 20um). (J-L), naïve animals. (J) Normal interstitial spaces (100x, size bar is 50um). (K) Normal bronchiolar epithelium (400x, size bar is 20um). (L) Normal tracheal mucosa (400x, size bar is 20um).

$10^{7}-10^{9} \mathrm{TCID}_{50}$ per gram of tissue, even at $24 \mathrm{dpi}$ (Figure 7(C)).

Histopathologic analysis of the lung sections of all evaluated IL2RG KO hamsters exhibited disseminated, moderate to severe, chronic-active interstitial pneumonia. Alveolar septa were expanded by moderate amounts of fibrin, variably well-organized bundles of collagen and infiltrated by moderate numbers of neutrophils and macrophages. Adjacent alveolar spaces frequently contained moderate numbers to numerous macrophages with fewer degenerate and non-degenerate neutrophils admixed with cellular debris (Figure $7(\mathrm{D})$ ). Greater than $50 \%$ of evaluated alveolar spaces were lined by type II pneumocytes that occasionally exhibited pseudo-stratified, columnar epithelial differentiation with a distinct ciliated apical border. Lymphocyte infiltrates and perivascular lymphoid aggregates were absent in all evaluated sections. Immunohistochemical reaction revealed numerous immunoreactive type I and type II pneumocytes as well as immunoreactive ciliated bronchiolar epithelial cells (Figure 7(E)). Histopathologic evaluation of the spleen confirmed the absence of lymphoid follicles and peri-arteriolar lymphoid sheaths. Extramedullary hematopoiesis, noted in both the spleen and liver, consisted entirely of erythroid lineage cell populations.

\section{Discussion}

SARS-CoV-2 infection in humans varies from asymptomatic to severe respiratory disease that can be fatal, especially in elderly and otherwise immunocompromised individuals. The lack of preclinical animal 
A

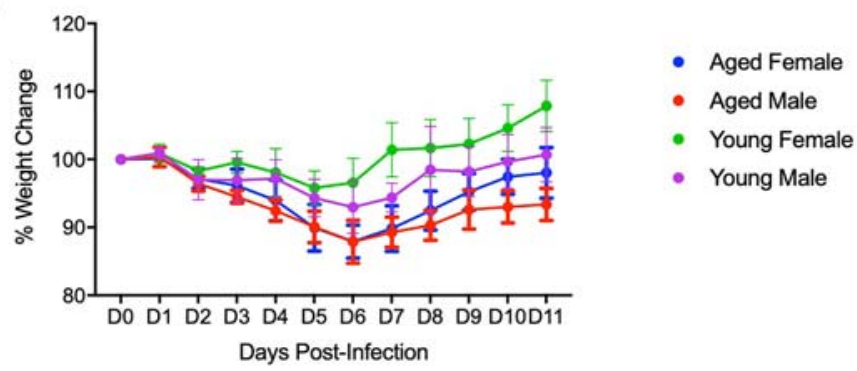

B

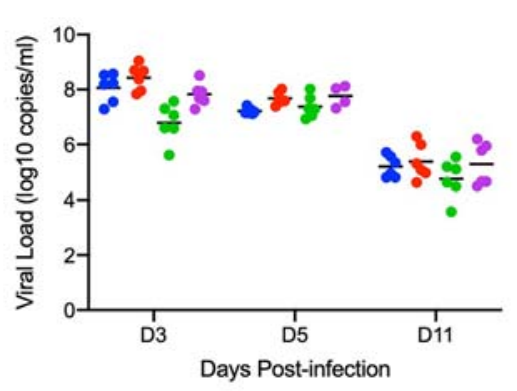

D

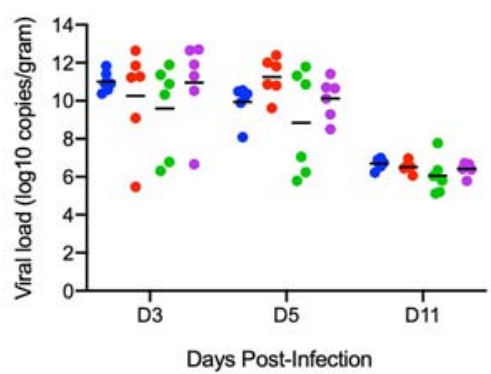

C

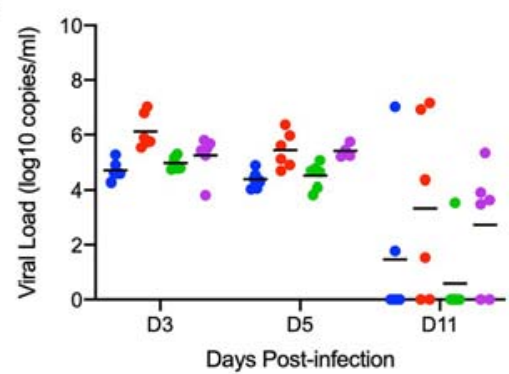

$\mathrm{E}$

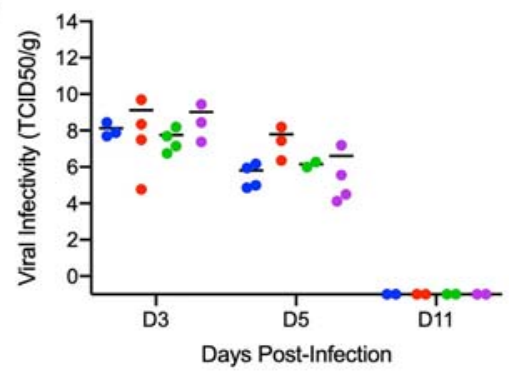

Figure 6. Neither age nor sex affects shedding or disease following infection with SARS-CoV-2. To compare the effects of aging and sex on disease following SARS-CoV-2 infection, young female and male (4-6weeks) and aged female and male (>6months) Syrian hamsters were infected intranasally with $500 \mathrm{ID}_{50}\left(10^{3} \mathrm{TCID}_{50}\right)$ of SARS-CoV-2. Samples were collected at the time points noted. Weights were collected daily, shedding and viral loads in the lungs were measured. (A) Daily weights. (B) Viral genome load recovered from oral swabs at each time point. (C) Viral genome load recovered from rectal swabs at each time point. (D) Viral genome load recovered from lungs at each terminal point. (E) Infectious titres in the lungs. ANOVA was used to compare groups at each time where samples were collected. No significant differences were observed between groups at any time point collected in in this study. Note: blue circles, aged females; red circle, aged male; green circle, young female; purple circle, young male.

models that replicate the severe disease of some COVID-19 patients is a substantial hurdle for the progression of promising countermeasures from in vitro testing through to clinical trials. A reliable small animal model would allow reproducible, in-depth analyses of infection patterns, elucidation of the immune response to SARS-CoV-2 infection and serve as a critical preclinical model for the initial in vivo step in evaluating COVID-19 countermeasures for human use.

The Syrian hamster has been established as a SARS-CoV-2 animal model, but a more thorough analysis had yet to be performed. To expand our understanding the Syrian hamster model, we first determined that the hamster ACE2 receptor was compatible with the SARS-CoV-2 spike protein binding domain. The level of binding and entry was consistently higher than the corresponding assay testing human ACE2 binding activity. As the receptor binding data suggested and other recent studies have shown, Syrian hamsters were susceptible to SARS-CoV-2 infection resulting in moderate to severe broncho-interstitial pneumonia and prolonged virus shedding of at least 10 days. The $\mathrm{ID}_{50}$ of SARS$\mathrm{CoV}-2$ in the Syrian hamster is low, roughly five infectious particles will result in a productive infection in $50 \%$ of animals.

Symptoms of disease appear approximately $3 \mathrm{dpi}$, but clinical signs are minimal with a consistent but not severe weight loss of 5-10\%. Ruffled fur may be observed in some hamsters, respiration rates may increase slightly, but the behaviour is unchanged from naïve hamsters. Infection was systemic following intranasal inoculation and viral gRNA was detected in all tissues examined. However, the lungs were the major site of viral replication and clearly 
A

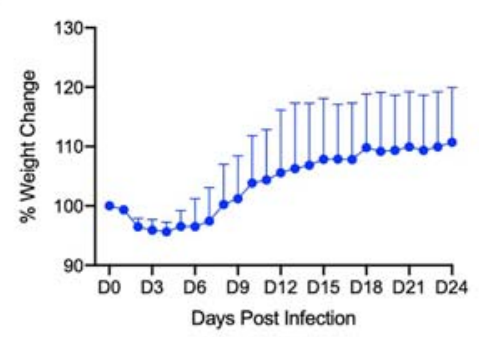

B

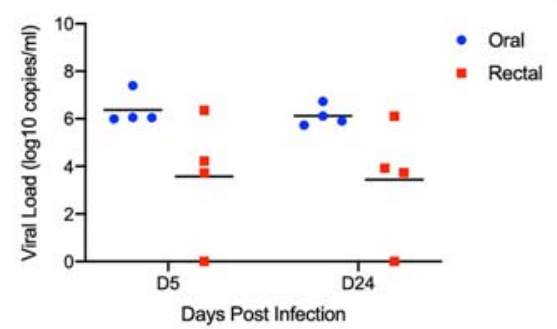

C

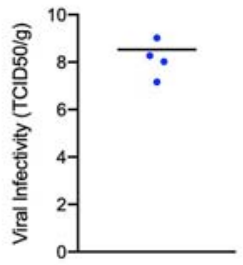

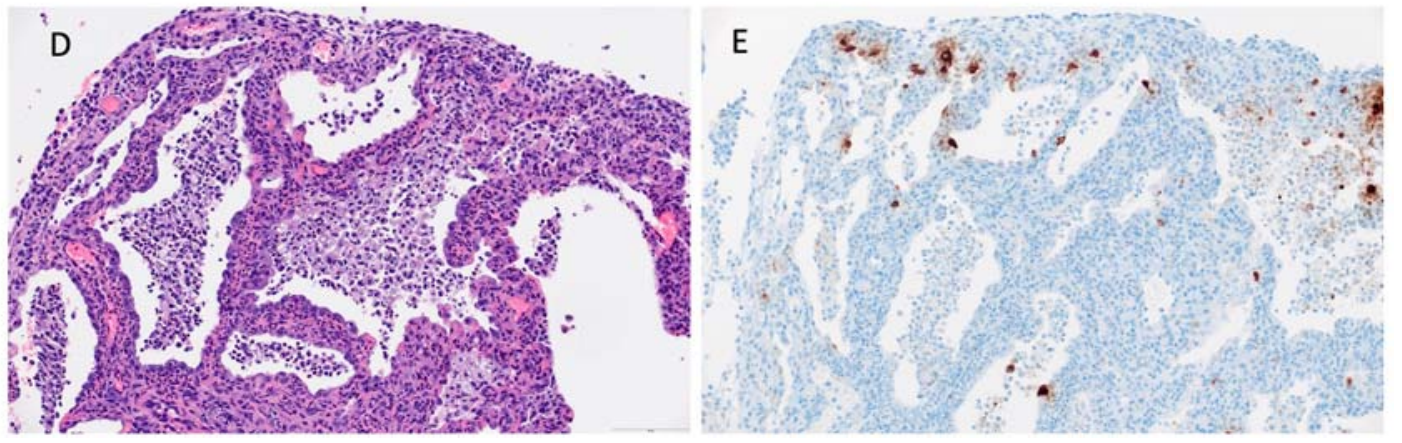

Figure 7. SARS-CoV-2 infection of Interleukin-2 receptor subunit gamma knockout hamsters $\left(I L 2 R G^{-1}\right)$ results in persistent infection and pneumonia. IL2RG KO hamsters lacking mature B-cells, T-cells and NK cells, were infected with $5 \times 10^{4} I_{50}\left(10^{\wedge} 5 \mathrm{TCID}_{50}\right)$ and followed for 24 days to determine if disease developed. Weights were collected daily and shedding from mucosal membranes and viral infectivity in the lungs were measured at the time points noted. (A) Daily weights. (B) Viral genome load recovered from oral and rectal swabs at each time point. (C) Infectious titres in the lungs. (D) Alveoli frequently contain macrophages, neutrophils and sloughed epithelial cells, and are lined by numerous hyperplastic type II pneumocytes (H\&E, 200x). (E) Immunoreactivity is observed in hyperplastic type II pneumocytes and macrophages (Anti-SARS-CoV-2 nuclear protein, 200x). Note (B): blue circles, oral swabs; red circle, rectal swabs. Size bar is 100 um.

showed a consistent but moderate pathology. Following intranasal infection, interstitial pneumonia initiating as bronchiolitis and focusing around terminal airways developed at $3 \mathrm{dpi}$ but was more severe in the animals receiving higher infectious doses at this early time point. Pulmonary pathology continued to increase in severity and extent of lesions in the lower dosed animals and was more severe at $5 \mathrm{dpi}$, with characteristic evidence of coronaviral infection noted including presence of syncytial cells in bronchioles and alveolar spaces. Pulmonary pathology was diminished by 10dpi in all animals examined at those times with characteristic evidence of epithelial regeneration noted.

In the human population, there have been reports of an increase in COVID-19 severity in males $[36,37]$ as well as a disparity of COVID-19 severity among different age groups $[38,39]$. In these studies, neither age nor gender was a factor in disease severity or outcome. Young hamsters had similar shedding kinetics, virus titres in the lungs and developed similar pulmonary pathology as aged hamsters regardless of sex. Importantly, all animals taken at the late timepoints showed evidence of recovery from disease at a similar rate.

Interestingly, elimination of host adaptive immune responses in the IL2RG KO model resulted in a chronic infection persisting at least 24 days. Virus was detectable in oral and rectal swabs at 5dpi and at study termination (24dpi). Histopathology evaluated at $24 \mathrm{dpi}$ supports a chronic-active infection of the respiratory system with foci of epithelial regeneration as well as active recruitment of neutrophils and macrophages. Unlike the immunocompetent hamster model, in which antigen was only detectable outside of regions of pathology, SARS-CoV-2 antigen was detectable in type I and type II pneumocytes, hyperplastic pneumocytes in regions of regeneration and macrophages. Additionally, viral antigen was present in morphologically normal bronchial epithelial cells at $24 \mathrm{dpi}$, a feature only routinely observed at $3 \mathrm{dpi}$ in the immunocompetent hamster model. While histopathology revealed a moderate to severe pulmonary inflammatory response, clinical signs of severe respiratory disease was not observed in this model. These data suggest that the innate immune system, in the background of compromised adaptive immunity, is capable of depressing the viral infection enough to keep respiratory physiology relatively stable, but incapable of eliminating SARS-CoV-2 infection. Additionally, vascular changes and perivascular leukocyte infiltrates were not observed in the IL2RG KO model, unlike the immunocompetent hamster model. This data, even though limited to four animals, suggests that the adaptive immune response or IL-2 signalling pathway may play a critical role in the 
development of innate leukocyte recruitment and staging during viral infection and the regulation of the coagulation cascade in response to a pro-inflammatory local environment.

Both autopsy and terminal biopsy samples from human patients exhibiting COVID-19 disease have shown histologic evidence of diffuse alveolar disease, and death is frequently attributed to the clinical progression of pneumonia, often times resulting in acute respiratory distress. Diffuse alveolar disease in SARS-CoV-2 infection has characteristic lesions of hyaline membrane formation in alveolar spaces accompanied with proteinaceous fluid leaking from damaged vessels into alveolar spaces. In hamsters, there is evidence of alveolar epithelial damage at peak virus replication with lesion resolution later on. However, the hamster model fails to develop fulminant diffuse alveolar disease and lacks the respiratory decompensation associated with the clinical syndrome of acute respiratory distress. Relative to the recently developed NHP models $[9,11]$, the Syrian hamster model exhibits a similar mild disease phenotype and is well suited for assessing therapeutics or vaccines.

Another effective small animal model of COVID19, the human ACE2 mouse, show these animals develop pneumonia resulting in fatal disease following SARS-CoV-2 infection $[16,26,40]$. However, with this mouse model there is concern about the location and level of receptor expression in these human ACE2 transgenic mice. The increase in expression locales and levels could result in enhanced or systemic disease dissimilar to COVID-19 in humans as these animals have been reported to develop fatal encephalitis [40], a clinical manifestation not currently associated with severe COVID-19 disease in humans. Additionally, the human ACE2 mouse has been previously shown to cause neuronal death without evidence of encephalitis in the SARS-CoV-1 model [41]. These complications may limit therapeutic studies in the human ACE2 mouse model.

Although, as with all animal models, there are some limitations exemplified by the lack of a systemic response to SARS-CoV-2 infection. The only mild disease manifestation and ability to quickly limit the infection make this model less suitable to study the mechanisms of severe COVID-19. However, the consistent and easily measured lung disease found in hamsters of all ages and sex make this a suitable infection model to evaluate SARS-CoV-2 countermeasure development.

\section{Acknowledgements}

The authors thank the animal caretakers and histopathology group of the Rocky Mountain Veterinary Branch (NIAID, $\mathrm{NIH}$ ) for their support with animal related work, and Anita Mora (NIAID, NIH) for help with the display items.

\section{Disclosure statement}

No potential conflict of interest was reported by the author(s).

\section{Funding}

The work was funded by the Division of Intramural Research, National Institutes of Allergy and Infectious Diseases (NIAID), National Institutes of Health (NIH), and partially funded through awards to The Vaccine Group Ltd, and the University of Plymouth.

\section{Disclaimer}

The opinions, conclusions and recommendations in this report are those of the authors and do not necessarily represent the official positions of the National Institute of Allergy and Infectious Diseases (NIAID) at the National Institutes of Health (NIH), Utah State University and University of Plymouth.

\section{ORCID}

Vincent Munster (iD http://orcid.org/0000-0002-2288-3196 Heinz Feldmann (i) http://orcid.org/0000-0001-9448-8227

\section{References}

[1] WHO Coronavirus Disease (COVID-19) Dashboard 2020 [Maps, Charts and Data]. Available from: https://covid19.who.int.

[2] CDC COVID Data Tracker. (2020). [cited 2020 September 23, 2020]. Maps, Charts and Data]. Available from: https://covid.cdc.gov/covid-datatracker/\#cases_casesinlast7days.

[3] Chen N, Zhou M, Dong X, et al. Epidemiological and clinical characteristics of 99 cases of 2019 novel coronavirus pneumonia in Wuhan, China: a descriptive study. Lancet. 2020;395(10223):507-513.

[4] Wang D, Hu B, Hu C, et al. Clinical characteristics of 138 hospitalized patients with 2019 novel coronavirusinfected pneumonia in Wuhan, China. JAMA. 2020.

[5] Gautret P, Lagier JC, Parola P, et al. Hydroxychloroquine and azithromycin as a treatment of COVID-19: results of an open-label non-randomized clinical trial. Int $\mathrm{J}$ Antimicrob Agents. 2020:105949.

[6] Sanders JM, Monogue ML, Jodlowski TZ, et al. Pharmacologic treatments for coronavirus disease 2019 (COVID-19): a review. JAMA. 2020;323 (18):1824-1836.

[7] Ali MJ, Hanif M, Haider MA, et al. Treatment options for COVID-19: a review. Front Med (Lausanne). 2020;7(480).

[8] Jarvis MA, Hansen FA, Rosenke K, et al. Evaluation of drugs for potential repurposing against COVID-19 using a tier-based scoring system. Antivir Ther. 2020.

[9] Munster VJ, Feldmann F, Williamson BN, et al. Respiratory disease in rhesus macaques inoculated with SARS-CoV-2. Nature. 2020.

[10] Finch CL, Crozier I, Lee JH, et al. Characteristic and quantifiable COVID-19-like abnormalities in CT- and PET/CT-imaged lungs of SARS-CoV-2-infected crabeating macaques (Macaca fascicularis). bioRxiv. 2020. 
[11] Rockx B, Kuiken T, Herfst S, et al. Comparative pathogenesis of COVID-19, MERS, and SARS in a nonhuman primate model. Science. 2020;368(6494):10121015.

[12] Kim YI, Kim SG, Kim SM, et al. Infection and rapid transmission of SARS-CoV-2 in ferrets. Cell Host Microbe. 2020;27(5):704-9.e2.

[13] Schlottau K, Rissmann M, Graaf A, et al. SARS-CoV-2 in fruit bats, ferrets, pigs, and chickens: an experimental transmission study. Lancet Microbe. 2020.

[14] Shi J, Wen Z, Zhong G, et al. Susceptibility of ferrets, cats, dogs, and other domesticated animals to SARScoronavirus 2. Science. 2020;368(6494):1016-1020.

[15] Dinnon KH, Leist SR, Schäfer A, et al. A mouseadapted SARS-CoV-2 model for the evaluation of COVID-19 medical countermeasures. bioRxiv. 2020:2020.05.06.081497.

[16] Bao L, Deng W, Huang B, et al. The pathogenicity of SARS-CoV-2 in hACE2 transgenic mice. Nature. 2020.

[17] Imai $M$, Iwatsuki-Horimoto $K$, Hatta $M$, et al. Syrian hamsters as a small animal model for SARS-CoV-2 infection and countermeasure development. Proc Natl Acad Sci U S A. 2020.

[18] Gu H, Chen Q, Yang G, et al. Rapid adaptation of SARS-CoV-2 in BALB/c mice: novel mouse model for vaccine efficacy. bioRxiv. 2020:2020.05.02.073411.

[19] Sia SF, Yan LM, Chin AWH, et al. Pathogenesis and transmission of SARS-CoV-2 in golden hamsters. Nature. 2020.

[20] Letko M, Marzi A, Munster V. Functional assessment of cell entry and receptor usage for SARS-CoV-2 and other lineage B betacoronaviruses. Nat Microbiol. 2020;5(4):562-569.

[21] Wan Y, Shang J, Graham R, et al. Receptor recognition by the novel coronavirus from Wuhan: an analysis based on decade-long structural studies of SARS coronavirus. J Virol. 2020;94(7):e00127-20.

[22] Hassan AO, Case JB, Winkler ES, et al. A SARS-CoV-2 infection model in mice demonstrates protection by neutralizing antibodies. Cell. 2020.

[23] Israelow B, Song E, Mao T, et al. Mouse model of SARS-CoV-2 reveals inflammatory role of type I interferon signaling. bioRxiv. 2020:2020.05.27.118893.

[24] McCray PB Jr, Pewe L, Wohlford-Lenane C, et al. Lethal infection of K18-hACE2 mice infected with severe acute respiratory syndrome coronavirus. J Virol. 2007;81(2):813-821.

[25] Tseng CT, Huang C, Newman P, et al. Severe acute respiratory syndrome coronavirus infection of mice transgenic for the human Angiotensin-converting enzyme 2 virus receptor. J Virol. 2007;81(3):1162-1173.

[26] Sun SH, Chen Q, Gu HJ, et al. A mouse model of SARS-CoV-2 infection and pathogenesis. Cell Host Microbe. 2020.

[27] Wang -J, Shuai -L, Wang -C, et al. Mouse-adapted SARS-CoV-2 replicates efficiently in the upper and lower respiratory tract of $\mathrm{BALB} / \mathrm{c}$ and $\mathrm{C} 57 \mathrm{BL} / 6 \mathrm{~J}$ mice. Protein Cell. 2020;11(10):776.

[28] Harcourt J, Tamin A, Lu X, et al. Severe acute respiratory syndrome coronavirus 2 from patient with 2019 novel coronavirus disease, United States. Emerg Infect Dis. 2020;26(6).

[29] Li R, Ying B, Liu Y, et al. Generation and characterization of an IL2RG knockout Syrian hamster model for XSCID and HAdV-C6 infection in immunocompromised patients. Dis Model Mech. 2020.

[30] Reed LJ, Muench H. A simple method of estimating fifty per cent endpoints12. Am J Epidemiol. 1938;27 (3):493-497.

[31] Corman VM, Landt O, Kaiser M, et al. Detection of 2019 novel coronavirus $(2019-\mathrm{nCoV})$ by real-time RT-PCR. Euro Surveill. 2020;25(3).

[32] Zhou P, Yang XL, Wang XG, et al. A pneumonia outbreak associated with a new coronavirus of probable bat origin. Nature. 2020;579(7798):270-273.

[33] Suresh V, Parida D, Minz AP, et al. Tissue distribution of ACE2 protein in Syrian golden hamster (Mesocricetus auratus) and its possible implications in SARS-CoV-2 related studies. bioRxiv. 2020:2020.06.29.177154.

[34] Leonard WJ, Lin JX, O'Shea JJ. The gammac family of cytokines: basic biology to therapeutic ramifications. Immunity. 2019;50(4):832-850.

[35] Allenspach E, Rawlings DJ, Scharenberg AM, et al. In: Adam MP, Ardinger HH, Pagon RA, Wallace SE, Bean LJH, Stephens K, editor. X-linked severe combined immunodeficiency. Seattle, WA: GeneReviews((R)); 1993.

[36] La Vignera S, Cannarella R, Condorelli RA, et al. Sexspecific SARS-CoV-2 mortality: among hormonemodulated ACE2 expression, risk of Venous Thromboembolism and Hypovitaminosis D. Int J Mol Sci. 2020;21(8).

[37] Conti P, Younes A. Coronavirus COV-19/SARS-CoV2 affects women less than men: clinical response to viral infection. J Biol Regul Homeost Agents. 2020;34(2).

[38] Grasselli G, Zangrillo A, Zanella A, et al. Baseline characteristics and outcomes of 1591 patients infected with SARS-CoV-2 admitted to ICUs of the Lombardy Region, Italy. Jama. 2020;323(16):1574-1581.

[39] Park SE. Epidemiology, virology, and clinical features of severe acute respiratory syndrome -coronavirus-2 (SARS-CoV-2; Coronavirus Disease-19). Clin Exp Pediatr. 2020;63(4):119-124.

[40] Jiang RD, Liu MQ, Chen Y, et al. Pathogenesis of SARS-CoV-2 in transgenic mice expressing Human Angiotensin-Converting Enzyme 2. Cell. 2020.

[41] Netland J, Meyerholz DK, Moore S, et al. Severe acute respiratory syndrome coronavirus infection causes neuronal death in the absence of encephalitis in mice transgenic for human ACE2. J Virol. 2008;82 (15):7264-7275. 